\title{
Saúde e bem-estar psicológico na(s) experiência(s) de pandemia
}

Health and psychological well-being in pandemic experience(s)

Carla Moleiro - ISCTE

carla.moleiro@iscte-iul.pt

doi: $x x x x x x x x x x x$

\begin{tabular}{|c|c|c|}
\hline Recebido / Received & Aceite / Accepted & Publicado / Published \\
27.09 .2020 & 16.10 .2020 & 1.06 .2021 \\
\hline
\end{tabular}

Como citar este capítulo / How to quote this chapter:

Moleiro, C. (2021). "Saúde e Bem-Estar Psicológico na(s) Experiência(s) de Pandemia". In Reis, B., Um mundo de incertezas; as leituras possíveis de um tempo pandémico (pp. 112-130). Lisboa: NIP-C@M \& UAL, disponível em xxxxxxxxx. DOI xxxxxxx. 


\section{Introdução}

Apesar de ter sido há (apenas) pouco mais de um ano que a presente pandemia global atravessou as nossas vidas, há muito tempo que conhecemos os efeitos psicológicos advindos de múltiplas das dimensões que passaram a caraterizar o nosso quotidiano. Incerteza. Medo. Isolamento. Ameaça. Perdas. Sabemos também muito sobre a importância do sentimento de segurança e de pertença, da rotina e da previsibilidade, das relações afetivas e do suporte social, do toque e do contato físico, do exercício e de brincar, da identidade social e da ligação com a comunidade.

O presente capítulo aborda os efeitos do medo e da incerteza no funcionamento psicológico, refletindo ainda sobre os impactos do isolamento social e da quarentena impostos pela realidade da pandemia. Identificam-se algumas variáveis protetoras da saúde e do bem-estar psicológico, continuando pela elaboração sobre a relevância das relações interpessoais e do contato físico e social a diversos níveis. Concluímos com a reflexão sobre os desafios específicos das pessoas em diversos grupos, em situações de vulnerabilidade, como estádios de desenvolvimento ao longo do ciclo de vida e grupos estigmatizados, bem como as disparidades de saúde resultantes ou agravadas pela pandemia.

\section{Sobre o medo e a incerteza}

O medo é uma emoção fundamental na experiência emocional e útil, hoje e ao longo da nossa história evolutiva, para a sobre- 
vivência (Barrett, Lewis \& Haviland-Jones, 2018; Bradley, 2003), tendo um papel muito relevante na resposta (inicial) à pandemia. Com efeito, o medo constitui uma emoção básica e comum na experiência humana (Barrett, Lewis \& Haviland-Jones, 2018). É uma emoção caraterística das respostas a situações de perigo iminente percebido, ou a estímulos que simbolizam o perigo. Estes podem ser entidades, objetos ou situações, que são percebidos como ameaçadores no presente ou no futuro (resposta antecipatória), para a vida ou integridade física, bem-estar, segurança, afeto ou aceitação, ou outra condição vista como tendo valor para o/a próprio/a. O medo de se ser contagiado com SARS-CoV-2 e de ficar doente, ou outros próximos de nós, ou até mesmo o que ser responsável por transmitir a doença a outros, passou a estar presente na vida de muitos/as. As experiências de medo podem ser de intensidades diversas; contudo, as respostas comportamentais típicas podem ser categorizadas em 3 formas principais, designadamente, evitamento/fuga, confronto/luta ou "congelamento" (fight or flight, or freeze). Estas respostas estão associadas a alterações fisiológicas (como o aumento do ritmo cardíaco ou libertação de cortisol), necessárias para a reação à situação. Apesar das manifestações comportamentais comuns, a experiência subjetiva do medo pode ser muito diversa, dependendo de cada indivíduo e do contexto. Deste modo, o medo do contágio, por exemplo, resultará em comportamentos e respostas emocionais heterogéneas, que irão desde estratégias de coping de confronto aquando das idas às compras no supermercado ou a restaurantes, ao evitamento de qualquer contato com o exterior, ou até mesmo à total negação do risco 
e recusa de usar máscara. Salienta-se que o medo, por vezes, acompanha-se de uma apreensão antecipatória de consequências negativas futuras sem ter, necessariamente, um estímulo desencadeador identificável - a ansiedade - e que é muitas vezes acompanhada de humor disfórico (e.g. tristeza, angústia, depressão) e/ou de uma ativação não-direcionada (e.g. pânico, ansiedade social) (Bradley, 2003; Greenberg \& Goldman, 2018), deixando de ser funcional e adaptativo.

Muitos foram os relatos do aumento do stress experienciado a partir do início da pandemia. As respostas psicológicas e comportamentais decorrentes estarão subordinadas à avaliação que cada indivíduo faz do estímulo stressor, designadamente, a pandemia. Segundo a Teoria Transacional do Stress proposta por Lazarus (1966; Lazarus \& Folkman, 1984), a resposta de cada indivíduo a um estímulo stressor dependerá, por um lado, de uma avaliação primária que o mesmo faz do estímulo e, por outro, de uma avaliação secundária face à situação. A avaliação primária refere-se ao significado que o indivíduo atribui ao evento e ao nível de gravidade antecipado relativamente a essa ameaça. Neste caso, no início da pandemia, por exemplo, as imagens do sistema de saúde em rutura no Norte de Itália e as notícias sobre quadros clínicos graves decorrentes de infeções pelo SARS-CoV-2 contribuíram sobremaneira para uma avaliação da situação bastante negativa e de elevada gravidade. Em contraste, os quadros ligeiros de infeção e a mudança das caraterísticas demográficas dos/as pacientes conduziu a uma avaliação de menor gravidade. A avaliação secundária, por seu turno, refere-se àquela que é feita dos recursos de coping 
que cada pessoa considera possuir para lidar com a fonte de stress. Dito de outro modo, até que ponto cada um/a se avalia como capaz para acionar estratégias de regulação emocional, comportamental e interpessoal para lidar com o surto pandémico. Deste modo, o stress sentido por cada pessoa será tanto maior quanto: (i) a mesma percecione o(s) fator(es) de stress como uma(s) ameaça(s) grave(s); (ii) e, simultaneamente, considere ter poucos recursos de coping para lidar adequadamente com a(s) fonte(s) de stress (Lazarus, 1966; Lazarus \& Folkman, 1984).

Quando as exigências do meio são excessivas e/ou o indivíduo é exposto prolongadamente a situações de stress (designadamente, numa crise de saúde pública, crise social e económica, de tempo incerto e intensidade elevada), falamos em stress crónico. Este tipo de stress contribui para a ativação do sistema de gestão de stress, no qual se inclui o eixo hipotálamohipófise-córtex adrenal (i.e., HPA, Hypothalamic-PituitaryAdrenal), que fica sob pressão. Tal implica uma maior libertação de glucocorticoides que pode levar ao desenvolvimento de disfunções ao nível metabólico, imunitário e psicológico (Uchino, Cacioppo \& Kiecolt-Glaser, 1996). O stress crónico pode, inclusive, levar a mudanças na atividade basal do eixo HPA e a mudanças ao nível da reatividade face ao stress de caráter duradouro. Deste modo, níveis elevados e prolongados poderão estar associados a risco acrescido de diversos tipos de problemas de saúde, como são exemplo as condições respiratórias, autoimunes, cardiovasculares, metabólicas e hormonais, para além de problemas de saúde mental. 


\section{Sobre o Isolamento Social, Quarentenas e Perdas}

Para fazer face à pandemia, ao nível global, a comunidade científica acelerou esforços, com um aumento do trabalho académico dedicado à COVID-19 e problemáticas associadas, e um investimento em novas formas de investigação (básica e aplicada) e consequente publicação. Um conjunto de revisões sistemáticas da literatura foram também realizadas, impulsionadas pela Organização Mundial da Saúde (OMS), para proporcionar informação robusta a decisores políticos e de saúde pública. Foi o caso do estudo dos efeitos do isolamento e da quarentena, adotados a nível individual (e.g. pessoas infetadas ou em vigilância ativa) ou a nível grupal em muitas comunidades e muitos países na(s) fase(s) de contenção dos contágios, ou mais seletivamente em fases posteriores da pandemia (Brooks et al, 2020). O isolamento em casa ou em equipamentos próprios é uma medida importante, ainda assim não é sem consequências, principalmente ao nível da saúde mental, como a revisão de Brooks e seus colegas deu conta. Num trabalho que reuniu os resultados de 24 estudos no âmbito de outras situações pandémicas (como SARS, Ébola, gripe equina, H1N1), com populações heterogéneas (como pacientes e profissionais de saúde, famílias com crianças, estudantes universitários, donos de cavalos), diversos efeitos psicológicos foram identificados na maioria dos estudos, incluindo sintomas de reação aguda de stress, irritabilidade e raiva, sintomas depressivos e exaustão emocional, medos de contágio e comportamentos de evitamento, stress pós-traumático, alterações do sono e queixas cognitivas 
(e.g. dificuldades de concentração e estados de confusão). A frustração e o tédio também foram exacerbados, especialmente em face à perda de liberdade e de rotinas, e perda de contatos físicos e sociais. Alguns dos estudos revistos compararam populações em quarentena ou isolamento com indivíduos sem essas medidas; outros ainda acompanharam longitudinalmente pessoas sob estas medidas. Para os grupos com experiência de isolamento ou quarentena foram encontrados efeitos logo após 9 dias e até períodos de follow-up de 3 anos. Moderadores destes efeitos foram apontados, tais como a duração do período de isolamento, a natureza (in)voluntária, o estigma, e a qualidade da informação transmitida. A saber, os períodos mais curtos e voluntários parecem trazer menores consequências negativas, e os benefícios de informação de saúde clara e rápida foram também documentados.

Globalmente, portanto, são encontradas alterações psico-emocionais resultantes e/ou típicas da experiência de um evento traumático nas respostas ao isolamento e quarentena. Acrescem a estas alterações os efeitos da perda social. Com efeito, por um lado, os impactos da solidão são reconhecidos atualmente como um problema grave e real de saúde pública. Quando comparada com outros fatores de risco para a doença e a mortalidade, "a solidão mata mais do que a poluição do ar, a obesidade, o consumo de álcool ou tabaco" (Lima, 2018). Por outro lado, pelo aumento do cansaço, irritabilidade e menor acesso a espaços individuais (quer físicos, quer simbólicos), o aumento do conflito familiar (relações tensas, violência domés- 
tica ou maus tratos de crianças) foi também reportado por organismos diversos (como a Comissão para a Cidadania e Igualdade de Género (CIG) e o Instituto de Apoio à Criança (IAC)). Estes elementos são igualmente agentes patogénicos, contribuindo para a deterioração da saúde, reconhecidos num modelo biopsicossocial da saúde e da doença (Engel, 1977, 1980). Isto é, a conflitualidade e intensificação das relações sociais dentro de casa constitui uma situação de vulnerabilidade para a saúde e o bem-estar emocional, em particular para populações que possam estar já em contextos de maior risco (e.g. existência de condição psiquiátrica anterior, crianças e jovens de grupos minoritários ou estigmatizados).

Resultou que a pandemia se tornou não só uma crise epidemiológica, mas também uma crise psicológica. A saúde mental destacou-se como uma prioridade, quer no quotidiano de cada um/a, quer nas medidas de saúde pública - com a criação de recursos especializados de informação pela Direção Geral de Saúde (DGS) e pela Ordem dos Psicólogos Portugueses (OPP), e de intervenção psicológica, com linhas de apoio no serviço nacional de saúde e no terceiro setor. Com o isolamento, a perda de familiares e amigos, mas também a instabilidade económica e o desemprego, diversos impactos na saúde psicológica da população surgiram e irão surgir ao longo dos próximos tempos (Holmes et al., 2020). Numa primeira linha, as reações normativas de resposta ao stress foram vivenciadas por muitos/as, por exemplo com cansaço, dificuldades do sono e ativação emocional; numa segunda linha, num grupo menor de pessoas, foram 
sentidas necessidades acrescidas que resultaram em sintomas de depressão, perturbações de ansiedade, consumo de álcool, ou mesmo burnout e suicídio; e a um terceiro nível, para quem detinha condições de saúde mental pré-existentes, verificou-se a possibilidade do agravamento de quadros clínicos e/ou experiências agudas de stress (ver estudo longitudinal recente na população holandesa; Pan et al, 2021). Já ao fim de um ano de pandemia por SARS-CoV-2, foi possível verificar, numa amostra de mais de 250,000 pacientes, que cerca de um terço das pessoas que tiveram COVID-19 desenvolveram, após 6 meses, algum quadro neurológico ou psiquiátrico, em comparação com controlos (Taquet et al, 2021), com especial saliência dos sintomas ansiosos e cognitivos. Para todos/as, as respostas de promoção da saúde mental serão heterogéneas e especializadas, para se ajustarem às diversas necessidades e também perfis desenvolvimentais (e.g. crianças e jovens, idosos) e psico-sociais.

\section{Dos Fatores de Risco aos Fatores Protetores}

Desenvolvemos uma vinculação forte a uma pessoa desde logo no início na nossa vida. Estamos "programados" para tal desde o nascimento (Bowlby, 1969/1982; Ainsworth \& Bowlby, 1991; Ainsworth et al., 1978) e esse espaço íntimo, de afeto, de segurança, de toque físico, de brincadeira, de aprendizagem, de autorregulação torna-se basilar no nosso desenvolvimento, personalidade e funcionamento ao longo da vida. Da capacidade para nos relacionarmos com os outros, de criarmos amigos, de nos apaixonarmos, de amar incondicionalmente, de colaborar 
num grupo, equipa ou comunidade, e até (para alguns/as) de ligação com uma força superior, criam-se inúmeros fatores protetores para a saúde. A evidência sobre a associação entre as relações interpessoais, e em particular o suporte social percebido, e os indicadores de saúde, incluindo bem-estar psicológico, é vasta (ver Lima, 2018; Berkman \& Kawachi, 2000). Esta evidência abrange populações diversas, distintos contextos e condições ao longo do ciclo de vida. Na situação pandémica, isso não é exceção. Apesar do distanciamento social, sabemos que manter a ligação com a nossa família e rede de amigos/ as, e receber/prestar suporte social (mesmo que virtual, em alguns casos) será um importante fator protetor do bem-estar e da saúde. Em desconfinamento, proliferam meios adicionais para a promoção do espaço social nas esplanadas e projetos como "Brincar de rua!" ${ }^{1}$. Uma preocupação especial colocase sobre o impacto nas crianças e no seu desenvolvimento. É bem reconhecido o valor intrínseco de brincar (Ginsburg, 2007) - mesmo antes da pandemia, a Academia Americana de Pediatria salientava que brincar estava em risco (Yogman, et al., 2018), e agora mais ainda.

Também o contato físico, sabemos desde as experiências pioneiras de Harlow (com macacos rhesus), é essencial para o estabelecimento da segurança e conforto na relação do bebé com um/a cuidador/a. O contato físico é importante tanto para as crianças em idade precoce, onde as conquistas desenvolvimentais estão ancoradas na promoção da confiança

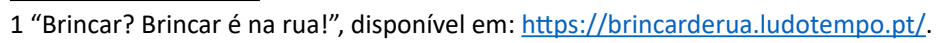


e na autonomia no conforto de (uma) relação segura (Teoria de Desenvolvimento Psicossocial de Erikson, 1980), como para as pessoas de idade avançada e as que se encontram doentes, para quem o toque pode, inclusivamente, ser visto como terapêutico; e todos os estádios de desenvolvimento entre uma fase e outra. Reinventou-se (e reinventa-se), por isso, o toque, que nos é tão essencial quando estamos com alguém com quem não pudemos estar durante meses de estado de emergência, ou aquando do desconfinamento das crianças para creches, jardins-de-infância e ATLs, ou quando confortamos alguém em luto face a uma perda - com cotovelos, abraços pela cintura ou pelas costas, com "bolhas" de contacto e brincadeira entre crianças da mesma sala de aula, entre pessoas que coabitam, ou mesmo nos próprios cuidados de saúde com luvas, máscaras e fatos de proteção integral.

Também a estrutura e a previsibilidade são basilares desde as referidas fases precoces da vida de uma criança, desde as reações circulares dos primeiros 2 anos de vida (estádio sensório-motor de Piaget) às rotinas diárias no contexto da escola e em casa. A criação de estrutura traz à criança alguma segurança na sua capacidade de prever e lidar com o seu meio ambiente, ao contrário da constante incerteza e imprevisibilidade. Na pandemia, por meio das restrições da saída de casa, perderam-se muitos aspetos da estruturação do quotidiano em teletrabaIho e em telescola, acrescendo-se a incerteza sobre a própria situação pandémica, a sua evolução ao longo do tempo e os seus impactos. Deste modo, benefícios foram reportados por 
aqueles/as que mantiveram uma estrutura diária e semanal, com o desenvolvimento de rotinas (e.g. em casa, a nível individual e em família) e estruturação de estratégias para organizar o funcionamento psicológico de crianças, jovens e adultos. Essas estratégias, apesar de previsíveis, puderam também trazer estimulação e novidade (e.g. maximizando as mais valias das tecnologias), bem como promover o nível de atividade física (e.g. corrida, surf, aulas coletivas de exercício físico online) - também elas necessárias ao bem-estar psicológico. Desenvolver e retomar antigas e novas rotinas no regresso às escolas, ao trabalho, aos espaços públicos e aos convívios sociais beneficiará o reforço das experiências de previsibilidade e de segurança, devolvendo a cada um/a o sentimento possível de controlo.

\section{Sobre as Desigualdades e as Identidades e Pertenças Sociais}

Ao contrário dos quadros clínicos apresentados na COVID-19, que têm uma causa muito específica e mecanismos determinados das respostas inflamatórias, nas perturbações no âmbito da saúde mental os fatores etiológicos são multi-dimensionais e complexos. Os determinantes incluem o indivíduo, com as suas facetas biológica e psicológica, bem como a interação deste com os seus diversos contextos sociais, designados no modelo ecológico de Bronfenbrenner $(1977,1989)$ como micro, meso, exo e macro-sistemas. Assim, de entre esses fatores salienta-se o papel o stress interpessoal e social, que ocorre em socie- 
dade, pela pertença a um determinado grupo. É reconhecido que as identidades sociais influenciam grandemente indicadores relativos à saúde (Drury et al., 2019; Tajfel \& Turner, 1979). Com efeito, as pertenças a grupos (e.g. ser mulher, migrante, idoso/a, LGBTQI+, Português) podem-se tornar identidades sociais, para além de experiências subjetivas e individuais. Essas identidades associam-se a significados, compreensões do/a próprio/a e dos outros, e a comportamentos. Podem, por isso mesmo, por um lado, constituir-se como veículos de acesso a recursos (individuais e coletivos) de apoio e estratégias de lidar com os desafios como a experiência da pandemia (e.g. o orguIho nacional no período pandémico do "milagre Português"; as manifestações de apoio a profissionais de saúde). Dito de outro modo, o grupo pode funcionar como um mecanismo de "cura social" ("Social Cure"; Jetten et al., 2017; Tajfel \& Turner, 1979).

Por outro lado, porém, a pertença a grupos minoritários, estigmatizados ou em condições de maior vulnerabilidade pode trazer também custos para a saúde, em particular a saúde mental (ver Berkman \& Kawachi, 2000). Pessoas de grupos minoritários ou estigmatizados têm mais probabilidade de manifestar um conjunto de condições de saúde, incluindo saúde mental, do que pessoas do grupo maioritário. Estas disparidades de saúde têm sido atribuídas quer a necessidades específicas destes mesmos grupos (às quais não são dadas respostas específicas ajustadas), quer (e principalmente) à vivência de formas únicas de stress por parte dos membros destes grupos, designadamente à experiência de diversas formas 
de violência interpessoal, discriminação, estigma e preconceito (e.g. stress minoritário em pessoas LGBTQI+, violência contra as mulheres e crianças, stress de aculturação de migrantes). Mais uma vez, também na pandemia estas evidências se encontram, traduzindo-se em disparidades de saúde documentadas (e.g. Chandan et al, 2020; Holmes et al., 2020). Foram diversos os dados que surgiram da maior gravidade dos quadros clínicos de pessoas com COVID-19, incluindo mortalidade, em membros de minorias étnicas em diversos países do mundo (e.g. comunidade afrodescendente e hispânica, incluindo profissionais de saúde).

A violência psicológica, física e sexual, em particular dentro de casa e dirigida a mulheres, crianças e jovens, foi reconhecida como uma realidade agravada pela situação de confinamento; e o custo do teletrabalho e da telescola na conciliação famíliatrabalho acentuado para as mulheres de um modo geral. Foi igualmente reconhecido o papel do acesso inadequado a serviços de saúde e da exclusão social na dificuldade que pessoas refugiadas e migrantes podem experienciar, com impactos na deteção precoce, testagem, diagnóstico, rastreio de contactos e prestação de cuidados de saúde face à COVID-19, aumentando o risco de surtos nestas comunidades. O mesmo se revelou para as populações indígenas (por exemplo, no Brasil, nos EUA, na Austrália). Também o estádio de desenvolvimento ao longo do ciclo de vida foi amplamente reconhecido como variável determinante nas experiências de pandemia. Crianças e jovens vivenciaram desafios únicos; o mesmo se reconhece para as pessoas de idade avançada - as/os avós e avôs. E na 
multiplicidade de dimensões e caraterísticas identitárias (ver intersecionalidade; Crenshaw, 1991) encontram-se as mulheres negras, os/as jovens LGBTQI+, as crianças refugiadas, as pessoas migrantes de idade avançada, e muitos outros. O estigma e o stress minoritário, de natureza crónica, constituirão determinantes do bem-estar, das condições de saúde e bem-estar psicológico de muitas pessoas, grupos e comunidades durante a evolução da crise de saúde pública, social e económica.

\section{Do Futuro da Saúde Mental e Bem-estar Psicológico}

Do mesmo modo que testemunhamos nos últimos tempos a aceleração científica da investigação sobre COVID-19 e áreas associadas, verificamos também a proliferação de respostas de apoio à população ao nível da saúde mental. $O$ reconhecimento da importância do bem-estar psicológico contribuiu para a maior visibilidade dos serviços disponíveis, mas acima de tudo para a destigmatização do tabu da saúde mental. Respostas diversas, incluindo consultas online e linhas gratuitas, foram surgindo. Relevantes stakeholders em Portugal (DGS, OPP) e no mundo (Associação Americana de Psicologia, APA; OMS) têm disponibilizado recursos online, muitos dirigidos à psicoeducação e prevenção, com informação clara, ajustada a diferentes públicos e temáticas (e.g. apoio aos próprios profissionais de saúde; intervenção com doentes recuperados de quadros clínicos graves de infeção por SARS-CoV-2; auxílio a famílias com crianças, entre outros). 
Mas a resiliência não se constrói apenas com ajuda clínica, de caráter mais individual; constrói-se também na promoção do suporte social e da partilha, e no apoio ao desenvolvimento de programas dirigidos a grupos e comunidades (Drury et al., 2019). Isso inclui o combate ao estigma e ao preconceito associado à COVID-19, incluindo a redução das barreiras experienciadas por populações mais vulneráveis ao acesso aos cuidados de saúde mental (Holmes et al., 2020), sensíveis/especializados e de qualidade - em resposta, por exemplo, à pobreza, ao stress minoritário, à violência interpessoal, às lacunas de formação específica dos profissionais e serviços. Também as ligações à comunidade ou a um grupo social promovem resiliência (Drury et al., 2019). O distanciamento social não significa isolamento social. E o (mais ou menos) prolongado período de elevados fatores de stress que se apresenta no futuro significa que temos de aumentar também o investimento nos fatores de proteção, aos níveis individual, familiar e grupal, e societal. Esse investimento deve ter como paralelo a investigação sobre as consequências não só da pandemia, mas da crise social e financeira, na saúde mental; e a procura das condições para mitigar os efeitos da pandemia na saúde mental e para dar respostas eficazes às necessidades dos grupos de maior vulnerabilidade (Holmes et al., 2020).

\section{Bibliografia}

Ainsworth, M. D. S., \& Bowlby, J. (1991). An ethological approach to personality development. American Psychologist, 46, pp. 331-341. 
Ainsworth, M. D. S., Blehar, M.C., Waters, E. \& Wall, S. (1978). Patterns of attachment. Lawrence Erlbaum Associates, Hillsdale, New Jersey.

Barrett, L. F., Lewis, M., \& Haviland-Jones, J.M. (2018). Handbook of Emotions ( $4^{\text {th }}$ edition). New York: Guilford.

Berkman, L. F. \& Kawachi, I. (2000). Social epidemiology. New York: Oxford University Press.

Bowlby, J. (1969/1982). Attachment and loss, Vol. 1: Attachment. New York: Basic Books.

Bradley, S. J. (2003). Affect Regulation and the Development of Psychopathology. New York: Guilford Press.

Bronfenbrenner, U. (1977). Toward an experimental ecology of human development. American Psychologist, 32, pp. 513-531.

Bronfenbrenner, U. (1989). Ecological systems theory. Annals of Child Development, 6, pp. 187-249.

Brooks, S. K., Webster, R. K., Smith, L. E., Woodland, L., Wessely, S., Greenberg, N., \& Rubin, G. J. (2020). The psychological impact of quarantine and how to reduce it: rapid review of the evidence, Lancet, 395, pp. 912-920. Doi: 10.1016/ S0140-6736(20)30460-8.

Chandan, J. S., Taylor, J., Bradbury-Jones, C., Nirantharakumar, K., Kane, E., \& Bandyopadhyay, S. (2020). COVID-19: A public health approach to manage domestic violence is needed. The Lancet Public Health, 2667(20), 30112, disponível em: https://doi.org/10.1016/s24682667(20)30112-2.

Crenshaw, K. (1991). Mapping the margins: Identity politics, intersectionality and violence against women. Stanford Law Review, 43, pp. 14-53.

Drury, J., Carter, H., Cocking, C., Ntontis, E., Tekin Guven, S. \& Amlôt, R. (2019). Facilitating collective psychosocial resilience in the public 
in emergencies: Twelve recommendations based on the social identity approach. Frontiers in Public Health, 7, 141. Doi: 10.3389/ fpubh.2019.00141.

Engel, G.L. (1977). The need for a new medical model: a challenge for biomedicine. Science, 196(4286), pp. 129-136.

Engel, G. L. (1980). The clinical application of the bio-psychosocial model. American Journal of Psychiatry, 137, pp. 535-544.

Erikson, E. H. (1980). Identity and the life cycle. New York: Norton \& Company.

Ginsburg, K. R. (2007). The importance of play in promoting healthy child development and maintaining strong parent-child bonds. Pediatrics, 119(1), pp. 182-191. Doi: 10.1542/peds.2006-2697.

Greenberg, L. \& Goldman, R. (2018). Clinical handbook of EmotionFocused Therapy. Washignton, DC American psychological Association.

Holmes, E. A., O'Connor, R. C., Perry, V. H., Tracy, I., Wessely, S., et al. (2020). Multidisciplinary research priorities for the COVID-19 pandemic: a call for action for mental health science. The Lancet Psychiatry, 7, pp. 547-560. Doi:10.1016/S2215-0366(20)30168-1.

Jetten, J., Haslam, S., Cruwys, T., Greenaway, K., Haslam, C. \& Steffens, N. K. (2017). Advancing the social identity approach to health and wellbeing: Progressing the social cure research agenda: Applying the social cure. European Journal of Social Psychology. Doi:10.1002/ejsp.2333.

Lazarus, R. S. (1966). Psychological stress and the coping process. New York: McGraw-Hill.

Lazarus, R. \& Folkman, S. (1984). Stress appraisal and coping. New York: Springer.

Lima, M. L. (2018). Nós e os outros: O poder dos laços sociais. Lisboa: Fundação Francisco Manuel dos Santos. 
Pan, K., Kok, A., Eikelenboom, M., Horsfall, M., Jörg, F., Luteijn, R.A., Rhebergen, D., van Oppen, P., Giltay, E.J., \& Penninx, B. (2021). The mental health impact of the COVID-19 pandemic on people with and without depressive, anxiety, or obsessive-compulsive disorders: a longitudinal study of three Dutch case-control cohorts. Lancet Psychiatry 8, pp. 121-29. Doi: 10.1016/ S2215-0366(20)30491-0.

Tajfel, H., \& Turner, J. (1979). An integrative theory of intergroup conflict. In: Austin WG, Worchel S, editors. The Social Psychology of Intergroup Relations (pp. 33-48). Monterey, CA: Brooks/Cole.

Taquet, M., Geddes, J.R., Husain, M., Luciano, S., \& Harrison, P.J. (2021). 6-month neurological and psychiatric outcomes in 236379 survivors of COVID-19: a retrospective cohort study using electronic health records. Lancet Psychiatry. Doi: https://doi.org/10.1016/ S2215$\underline{0366(21) 00084-5 .}$.

Uchino, B. N., Cacioppo J. T., \& Kiecolt-Glaser, J. K. (1996). The relationship between social support and physiological processes: A review with emphasis on underlying mechanisms and implications for health. Psychological Bulletin, 119, pp. 488-531. Doi: 10.1037/00332909.119.3.488.

Yogman, M., Garner, A., Hutchinson, J., Hirsh-Pasek, K., \& Golinkoff, R. M. (2018). The power of play: A pediatric role in enhancing development in young children. Pediatrics, 142(3), pp. 1-17. Doi: 10.1542/peds.2018-2058. 